\title{
Cambios históricos en el patrón del paisaje agrícola en un sistema de huerta tradicional de la provincia de Alicante: el caso de la Finca Lo Comptador ${ }^{1}$
}

\section{Historical changes in agricultural landscape patterns in a traditional irrigated system of Alicante province: the case of Lo Comptador property}

\author{
J. E. Martínez Pérez ${ }^{*}$ A. Belda Antolí ${ }^{* *}$, C. Martín Cantarino*** \\ y E. Seva Román**
}

\section{INTRODUCCIÓN}

Los paisajes son creaciones antrópicas que resultan de las transformaciones históricas llevadas a cabo por el hombre sobre el territorio, así como por la influencia de diferentes modelos socioeconómicos de intervención humana. En un momento dado, algunos de estos elementos más persistentes deben ser vistos como herencia histórica y solo puede explicarse su presencia en el paisaje a través de un análisis histórico detallado (Turri, 2002, p. 104). De esta forma, según González Bernáldez (1981, p. 17), la combinación de documentación

\footnotetext{
${ }^{1}$ El desarrollo de este estudio ha sido realizado gracias a la financiación por parte del proyecto de investigación WADI (INCO2003-MPC2-015226) de la Comisión Europea.

* Unidad de Cartografía de los Recursos Naturales, Universidad de Alicante. España (je.martinez@ua.es).

** Departamento de Ecología, Universidad de Alicante. España.

*** Instituto Multidisciplinar para el Estudio del Medio (IMEM), Universidad de Alicante. España.
} 
histórica y la percepción remota pueden ser de gran utilidad para comprender un determinado paisaje en la actualidad.

La unidad espacial de la actividad humana sobre el territorio se ha fijado tradicionalmente a través de las propiedades inmobiliarias de los registros catastrales. Su configuración espacial responde a una determinada estrategia guiada y constreñida por una serie de condiciones socio-históricas o biofísicas con un mayor nivel de organización o análisis. Un conocimiento exacto de los cambios históricos en la estructura del paisaje debe facilitar y proporcionar interpretaciones acerca del estado actual y futuro del paisaje (Bender, Boehmer, Jens y Schumacher, 2003, p. 112). Esto es particularmente valioso para una planificación más efectiva del paisaje a escala local, donde los cambios del paisaje son importantes para planificar la conservación (Cots-Folch, MartínezCasanovas y Ramos, 2009, p. 1).

Los estudios históricos de paisajes rurales se han basado tradicionalmente en la rica información contenida en fuentes cartográficas donde se reflejaba la distribución de fincas y parcelarios. Algunos de los trabajos clásicos basados en este tipo de información son los de Bloch (1931) en Francia, Sereni (1961) en Italia o Rackham (1986) en Gran Bretaña. Generalmente, los trabajos de este tipo han recurrido a determinados niveles de estudio impulsados por la necesidad de definir los límites de las propiedades individuales, así como la disponibilidad de cartografía catastral. El grado de detalle de estos mapas puede ser considerado demasiado alto, pudiendo contener informaciones muy detalladas y precisas, no solo de los límites de las explotaciones, sino también de la configuración espacial de las parcelas cultivadas y las no cultivadas (Faus Prieto, 1995, p. 211).

Otros estudios como los de Mometto (1992) o Turri (2002) se centran en el análisis diacrónico de fincas individuales mostrando la actual configuración de los paisajes mediterráneos. Como una consecuencia de los procesos sociopolíticos en la provincia de Alicante se establecieron formas de explotación agraria ligadas a sistemas de colonización agrícola. En este caso, la nueva estructura del paisaje resulta del drenaje de antiguos marjales con objeto de colonizar tierras degradadas que estaban sometidas a periódicos brotes de paludismo (Canales y Vera, 1985, p. 155).

Aunque existe una larga tradición de estudios del paisaje, desde un punto de vista histórico o geográfico, el desarrollo de novedosas técnicas en ecología del paisaje, especialmente desde los años 1980, permite disponer de un rico conjunto de posibles aproximaciones sobre el territorio (Lesschen, Cammeraat y Nieman, 2008, p. 1574). Este trabajo presenta los resultados preliminares que nacen a través de la integración de un conjunto de análisis históricos y 
ecológicos, discutiendo problemas metodológicos y poniendo de manifiesto la oportunidad que supone el uso de información espacial para el análisis de la diversidad territorial.

La finca de Lo Comptador limita al sur con el Parque Natural del Hondo, siendo producto de los mismos procesos de transformación que condujeron a la fundación de Carrizales en Elche, como parte importante de los nuevos territorios agrícolas recuperados a partir de antiguos marjales (Canales y Vera, 1985, p. 144). Esta explotación recibe el nombre del hecho de ser una propiedad del asistente o contador del Duque de Arcos y Maqueda, Marqués de Elche, destacado señor feudal y promotor del drenaje y recuperación de este antiguo marjal en la primera mitad del siglo XVIII (Box, 1987, p. 289).

El estudio se basa en el análisis de la evolución de este paisaje agrícola, desde la fecha de su creación por la bonificación de los antiguos marjales, como un ejemplo significativo del área de Carrizales, considerado actualmente como humedal e incorporado en la zona de protección de los parques naturales de El Hondo y Salinas de Santa Pola.

Este trabajo presenta el análisis de un sistema de huerta tradicional, teniendo en cuenta los cambios históricos en los usos del suelo. Este proyecto comienza con el acuerdo entre la Universidad de Alicante y la Comunidad de Regantes de Carrizales (Elche), en el año 2007, bajo el auspicio del proyecto WADI.

\section{ÁREA DE ESTUDio}

La zona objeto de estudio se sitúa en la parte meridional de la comarca del Bajo Vinalopó, en su transición al Bajo Segura, al sur de la provincia de Alicante, y en el área de influencia de los Parques Naturales de El Hondo y de las Salinas de Santa Pola, quedando delimitada por las coordenadas UTM 30N: 697000, 4191000; 699000, 4225000 (figura 1).

El ombrotipo de la zona de estudio es mediterráneo semiárido (Rivas-Martínez y Arnaiz, 1983), con una temperatura media anual de $18{ }^{\circ} \mathrm{C}$, presentando dos períodos diferenciados, uno con fuertes lluvias torrenciales en la estación de otoño-invierno y otro muy seco en verano y con precipitaciones medias anuales que no superan los $350 \mathrm{~mm}$. El índice de termicidad indica que dentro del bioclima Mediterráneo xérico oceánico, esta zona pertenece al termotipo Termomediterráneo (Pérez-Cueva, 1994, p. 140).

Tradicionalmente, los usos del suelo de esta región incluyen cultivos de regadío y actividades industriales con una alta diversidad de productos agrícolas, resultando en un rico paisaje cultivado. Recientes cambios en el paisaje, 
FIGURA 1

DETERMINACIÓN DEL ÁREA DE ESTUDIO, CON REFERENCIA GENERAL UTM 30N ED50, SOBRE LA PROVINCIA DE ALICANTE. (A) LOCALIZACIÓN GEOGRÁFICA DE LA FINCA LO COMPTADOR. (B) DETALLE DE LOS LÍMITES HISTÓRICOS DE LA FINCA LO COMPTADOR (1883)

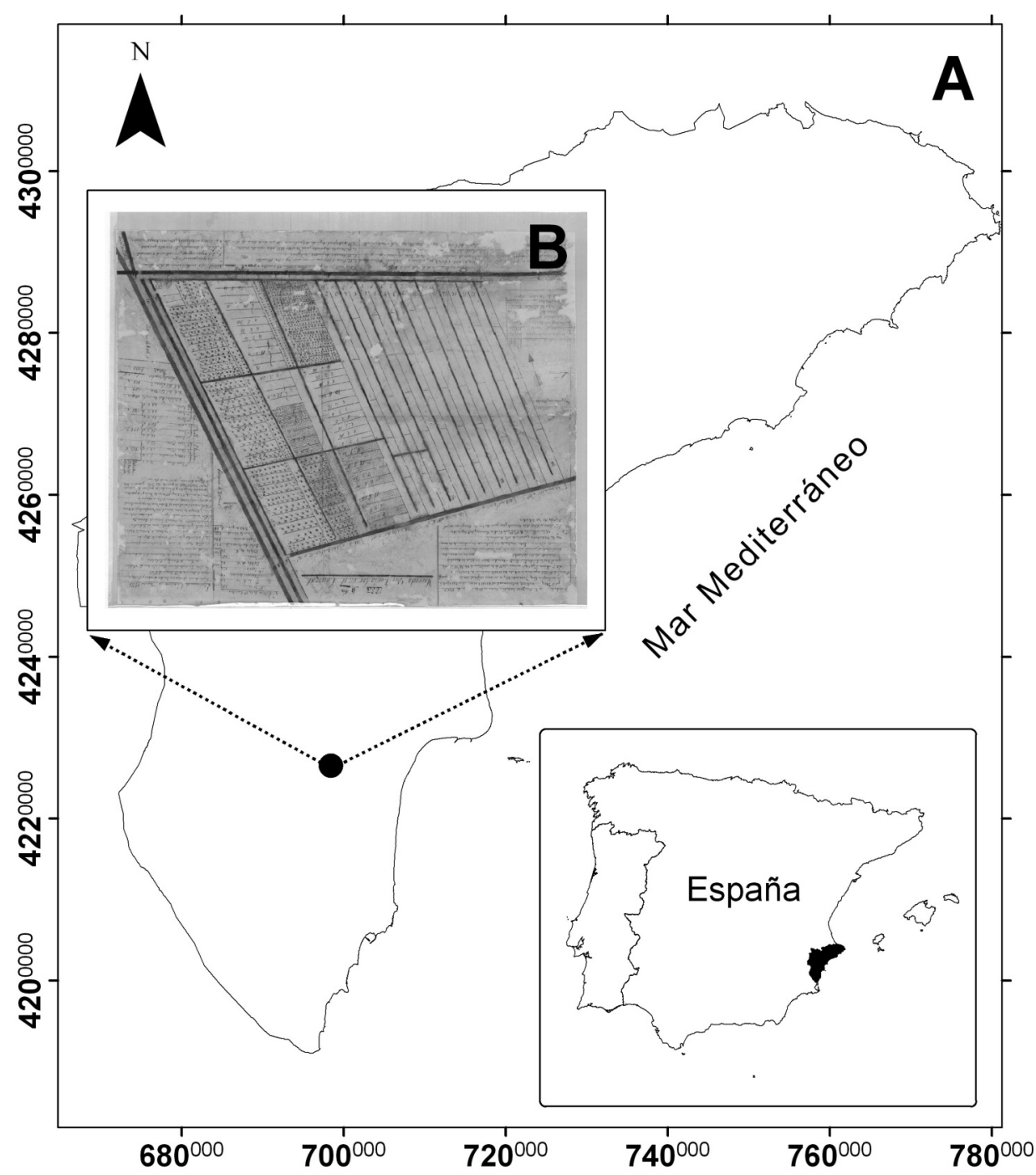


derivados de una pérdida de productividad, han conducido a una pérdida de diversidad paisajística debido a procesos de homogeneización en la estructura del paisaje, lo que puede suponer algunas repercusiones negativas sobre el balance ecológico de la zona.

\section{MATERIAL Y MÉTODOS}

El análisis de cambio de usos del suelo ha sido llevado a cabo mediante la utilización de sistemas de información geográfica. Este estudio se basa en una amplia variedad de fuentes, incluyendo mapas históricos y fotografías aéreas. Es fundamental recurrir a fuentes históricas, como la cartografía utilizada, ya que representa la estructura del paisaje tradicional, convirtiéndose en el punto de partida para el análisis de cambio del paisaje. La continuidad de un registro histórico a través de material gráfico, como el de las fotografías aéreas, es de gran importancia para este tipo de aproximaciones. De este modo, es posible considerar múltiples períodos de tiempo que representan la evolución de la estructura paisajística.

La utilización de cartografía antigua presenta una serie de dificultades a la hora de llevar a cabo la fotointerpretación, con lo cual se ha tenido que realizar una restitución y ajustar dicha cartografía a la imagen real, de este modo se ha empleado la ortoimagen georreferenciada del Instituto Cartográfico Valenciano (ICV) como referencia para llevar a cabo el estudio a nivel de usos del suelo.

Los mapas de usos del suelo, utilizados como base del estudio, fueron preparados mediante digitalización de las fotografías aéreas y los mapas históricos, una vez corregidas las deformaciones de este tipo de fuentes. Se obtuvieron los usos del suelo para los años 1883 (mapa histórico), 1956, 2002 y 2007 (fotografías aéreas). Se utilizaron los programas Cartalinx y ArcGis para crear estas cartografías.

A continuación se citan las diferentes fuentes cartográficas utilizadas para llevar a cabo el análisis de cambio:

\section{Levantamiento cartográfico de la Finca Lo Comptador del año 1883}

Se trata del típico mapa de agrimensor (Faus Prieto, 1995), coloreado en acuarela. No se tienen noticias del motivo por el que fue dibujado ni de las memorias a las que podría acompañar, aunque cabe suponer que se realizara con motivo del algún pleito sobre propiedad o exención de pago de censos. Indica con notable detalle, no sólo los elementos que delimitan la finca, sino 
su sistema interno de regadío y drenaje y la extensión y cultivos existentes de cada veleta o cuadra interna de la finca.

\section{Fotografía aérea 1956 (Vuelo Americano)}

Fotografía aérea de referencia a nivel nacional al tratarse del primer vuelo fotogramétrico que cubría la totalidad de la superficie del Estado Español.

\section{Ortoimagen del año 2002 del Instituto Cartográfico Valenciano (ICV)}

Ortofotograma del vuelo realizado por el Instituto Cartográfico Valenciano en formato digital en el año 2002 en el ámbito de la Comunidad Valenciana.

\section{Ortoimagen del año 2007 del Instituto Cartográfico Valenciano (ICV)}

Ortofotograma del vuelo realizado por el Instituto Cartográfico Valenciano en formato digital en el año 2007 en el ámbito de la Comunidad Valenciana.

Para llevar a cabo la creación de los mapas de usos del suelo se ha realizado una tarea de interpretación, en el caso del mapa histórico, y de fotointerpretación, sobre las fotografías aéreas, determinando las diferentes tipologías de cultivos presentes en la distribución parcelaria de la finca. Así, se han establecido un total de 15 elementos entre cultivos y estructuras, calculando la superficie que representan para cada uno de estos períodos.

\section{RESUlTAdos}

La figura 2 presenta la vista del área de estudio en las cuatro fechas consideradas para el análisis de cambio. Se ha utilizado un patrón geométrico específico para representar las categorías de usos del suelo en las cuatro instantáneas.

La totalidad de la finca cubre una superficie aproximada de 115 ha, manteniendo la estructura del parcelario a lo largo del tiempo y produciéndose un reemplazo de los usos del suelo dependiendo de las necesidades de cada momento. Actualmente, la dedicación de las tierras se centra, casi en exclusiva, a 


\section{FIGURA 2}

DISTRIBUCIÓN DE LAS CATEGORÍAS DE USOS DEL SUELO Y ESTRUCTURA PARCELARIA (PARA FACILITAR LA INTERPRETACIÓN SE HAN ELIMINADO LAS CATEGORÍAS «CASA»Y «ERA», DEBIDO A SU ESCASA ENTIDAD, REAGRUPANDO LAS CATEGORÍAS «LÍMITES» Y «MÁRGENES»)

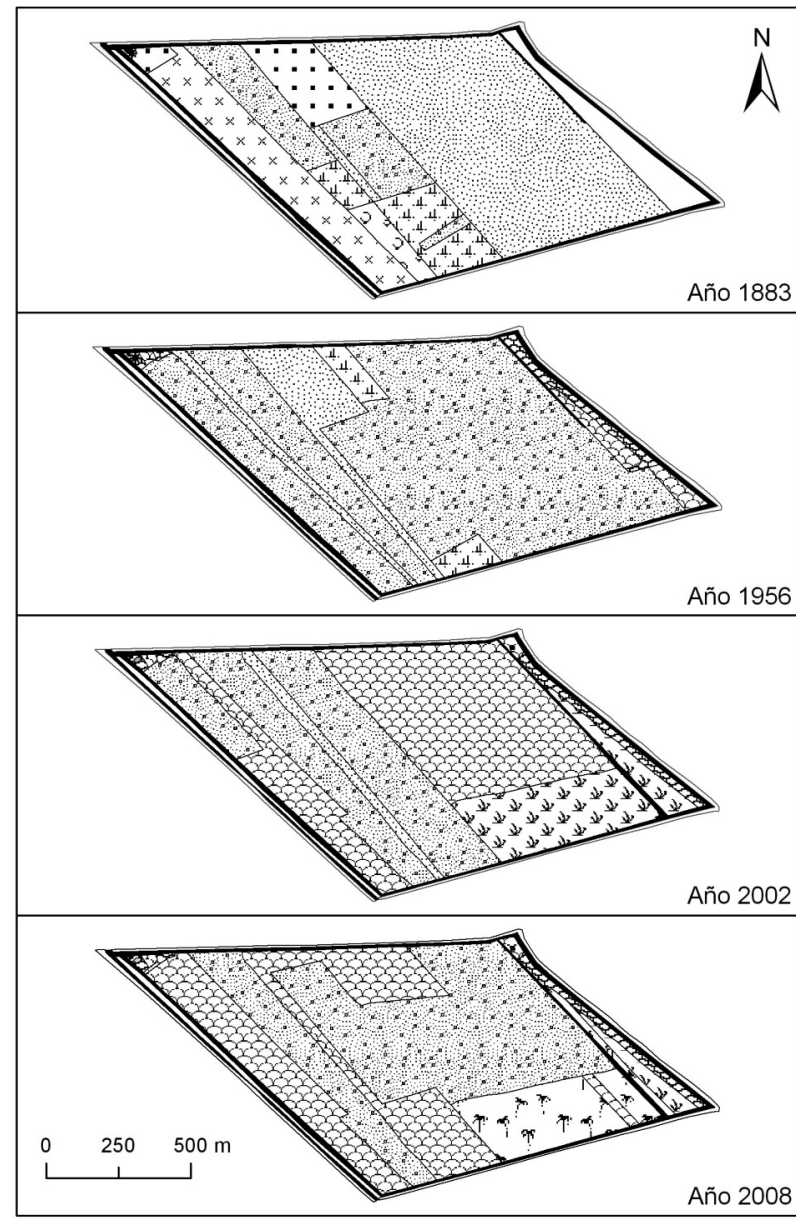

Leyenda

\begin{tabular}{|c|c|c|c|c|c|}
\hline & Límites y márgenes & {$\left[\begin{array}{lll}x & x & x \\
x & x & 1\end{array}\right]$} & Olivo adulto & $1 \%$ & Cebada \\
\hline & Canal de riego & & Olivo joven & & Alfalfa \\
\hline$x^{2}$ & Abandono reciente & $\theta^{\circ}$ & Olivo y viña & & Hortalizas \\
\hline 5 & Abandono antiguo & {$\left[\begin{array}{ll}4 \\
1\end{array}\right]$} & Viña & 17 & Palmera \\
\hline
\end{tabular}


los cultivos de cebada y algunas hortalizas. Esto se debe a la baja calidad de las aguas de riego que condicionan la implantación de cultivos resistentes a la salinidad. También cabe destacar la introducción de cultivos de palmera que son más rentables económicamente. Por último, es importante poner de manifiesto el abandono de buena parte del parcelario (30\%), lo que supone una situación comprometida frente a los procesos ecológicos que se desarrollan en el lugar.

El análisis visual de las capas obtenidas (figura 2) ilustra un alto grado de cambio en el paisaje en el período de investigación. Dentro de este mismo período, el olivar ha sido transformado hacia tierras abandonadas, mientras que áreas con viñedos se han convertido en cultivos de cebada y, parcialmente, también han sufrido un cierto proceso de abandono. La mayor pérdida de tierras agrícolas se ha producido en las parcelas de alfalfa, pasando de un $43 \%$ en el año 1883 a una situación en la que este cultivo es inexistente, debido a que en las últimas décadas ha desaparecido el uso de animales de carga en todas las actividades agrícolas. Por esta razón, esta categoría agrícola desaparece del paisaje agrario tradicional. Por otro lado, nuevos elementos, como las palmeras, han aparecido en el escenario local, dando respuesta a nuevas demandas de plantas mediterráneas ornamentales, alcanzando un $12 \%$ de la superficie del parcelario.

\section{CONCLUSIONES}

El análisis comparado de documentación cartográfica antigua a escala de finca y de fotogramas aéreos permite, pese a las considerables diferencias del material de base, un análisis diacrónico útil de los elementos del patrón espacial. Este análisis revela rasgos muy interesantes para la comprensión del paisaje de nuestros días. Este estudio ha permitido realizar una aproximación a nivel de parcela lo que puede suponer la integración de la información generada dentro de nuevas estrategias para la planificación del paisaje rural a escala local.

El uso de cartografía antigua y fotografía aérea proporcionan interesante información relacionada con los factores económicos, sociales y culturales del momento en que se generaron dichos documentos, pudiendo ser utilizados para desarrollar explicaciones sobre los cambios en el patrón paisajístico y crear adecuados escenarios de futuro.

El análisis de cambio de usos del suelo, basado en un SIG diacrónico, se ha definido como un instrumento valioso, especialmente para la cuantificación y 
TABLA 1

CAMBIOS DE USOS DEL SUELO PRODUCIDOS ENTRE 1883 Y 2008, CUANTIFICANDO LA SUPERFICIE DE CAMBIO (M²) EN LAS 15 CATEGORÍAS DE USOS DEL SUELO IDENTIFICADAS DURANTE EL PERÍODO DE ESTUDIO. LAS COLUMNAS PROPORCIONAN EL CAMBIO TEMPORAL (1883-2008) EN EL PATRÓN DEL PAISAJE AGRÍCOLA DE LA ZONA DE ESTUDIO

\begin{tabular}{lrrrr}
\hline Uso del suelo $\left(\mathrm{m}^{2}\right)$ & \multicolumn{1}{c}{1883} & \multicolumn{1}{c}{1956} & \multicolumn{1}{c}{2002} & \multicolumn{1}{c}{2007} \\
\hline Casa & 69,96 & 69,96 & 446,71 & 446,71 \\
Olivo adulto & $149.033,36$ & 0,00 & 0,00 & 0,00 \\
Olivo con viña & $25.216,65$ & 0,00 & 0,00 & 0,00 \\
Olivo joven & $76.646,62$ & 0,00 & 0,00 & 0,00 \\
Viña & $84.609,30$ & $40.530,73$ & 0,00 & 0,00 \\
Cebada & $137.902,58$ & $811.887,11$ & $343.201,29$ & $499.889,33$ \\
Alfalfa & $490.093,00$ & $117.302,36$ & $35.499,75$ & 0,00 \\
Hortalizas & 0,00 & 0,00 & $168.511,80$ & $21.193,88$ \\
Era & 335,05 & 335,05 & 335,05 & 335,05 \\
Límites & $48.946,71$ & $48.946,71$ & $48.946,71$ & $48.946,71$ \\
Canal de riego & $66.098,08$ & $66.098,08$ & $71.115,91$ & $71.115,91$ \\
Margen & $75.924,75$ & $17.574,33$ & $17.574,33$ & $17.574,33$ \\
Abandono reciente & 0,00 & $29.145,55$ & $445.166,26$ & $331.448,25$ \\
Abandono antiguo & 0,00 & $22.986,19$ & $24.078,25$ & $24.078,25$ \\
Palmera & 0,00 & 0,00 & 0,00 & $139.847,65$ \\
TOTAL & $1.154 .876,06$ & $1.154 .876,06$ & $1.154 .876,06$ & $1.154 .876,06$ \\
\hline
\end{tabular}

descripción del cambio en la estructura del paisaje a escala local. La aproximación cuantitativa del SIG, utilizada para explicar el cambio histórico del paisaje, es un componente esencial para la predicción de futuros cambios en el paisaje.

La finca Lo Contador, a través de este análisis preliminar, muestra una considerable permanencia de sus descriptores paisajísticos. Puede considerarse, por ello, un relicto histórico (como otras estructuras espaciales de la zona) del patrón territorial del siglo XviII impuesto por la desecación de los antiguos marjales.

Esta constancia parece deberse ante todo a los condicionantes impuestos por la red hídrica de irrigación y drenaje, que actúan como armazón persistente del resto de elementos paisajísticos. Las modificaciones introducidas a lo largo del tiempo (cambios de toma de aguas, canalizaciones, elevaciones, etc.) se han hecho amoldándose al sistema inicial. Puede hablarse, por tanto, de un patrón espacial del regadío, derivado de la forma espacial impuesta a los flujos hídricos desde el momento de la desecación y colonización de las antiguas marismas. 
Dentro de los planes de actuación programados para la reactivación de la economía local, se ha considerado la recuperación de las variedades de viña tradicional en el entorno de estos humedales. Esto puede suponer un revulsivo para la puesta en valor de un sistema agrícola en decadencia, que pasó de una situación de fuerte dependencia humana y alta productividad, a un estado de declive productivo y abandono de buena parte de la actividad antrópica. La recuperación de la actividad agrícola puede suponer la introducción de modificaciones interesantes en la estructura del paisaje, rompiendo con la homogeneidad estructural del abandono (Pausas, 2003, p. 365) y aportando una heterogeneidad ambiental que puede favorecer la colonización por parte de nuevos elementos de la fauna y flora locales.

\section{Agradecimientos}

Agradecemos la participación de todos los informantes locales, muchos de ellos vinculados a la Comunidad de Regantes de Carrizales (Elche), así como a los colaboradores de Dolores, la compañía Nuevos Riegos El Progreso y a todas y todos aquellos que hacen de la tierra su vida. Además, queremos expresar la ayuda prestada por la Dirección y el personal del Parque Natural del Hondo y del Parque Natural de las Salinas de Santa Pola. Así como también, a la dirección del Museo Escolar de Puçol y al Archivo Histórico de Elche, en especial a su director, D. Rafael Navarro Mallebrera y a la técnica Carmina Verdú.

Recibido: 01/06/2009

Aceptado: 20/09/2010

\section{BIBLIOGRAFÍA}

Bender, O., Boehmer H. J., Jens, D. y Schumacher, K. P. (2003): "Using GIS to analyse long-term cultural landscape change in Southern Germany". Landscape and Urban Planning, 70 (1-2), pp. 111-125.

Bloch, M. (1931): Les caractères originaux de l'histoire rurale française. Oslo, Institute pour l'Étude Comparative des Civilisations.

Box, M. (1987): Humedales y áreas lacustres de la provincia de Alicante. Alicante, Instituto de Estudios Juan Gil-Albert, Diputación Provincial de Alicante.

Canales, G. y Vera, J. F. (1985): "Colonización del cardenal Belluga en las tierras donadas por Guardamar del Segura: creación de un paisaje agrario y situación actual". Investigaciones Geográficas, 3, pp. 143-160. 
Comunidad de Regantes de Carrizales de San Francisco de Asís de la ciudad de Elche (1923): Ordenanzas de la Comunidad. Reglamento del Sindicato. Reglamento del Jurado. (ley de 25 de junio de 1885). Elche, Imprenta y Librería de José Agulló Sánchez.

Cots-Folch, R., Martínez-Casasnovas, J. A. y Ramos, M. C. (2009): "Agricultural trajectories in a Mediterranean mountain region (Priorat, NE Spain) as a consequence of vineyard conversion plans". Land Degradation and Development, 20, pp. 1-13.

Faus Prieto, A. (1995): Mapistes. Cartografia i agrimensura a la València del segle XVIII. Valencia, Edicions Alfons el Magnànim.

González Bernáldez, F. (1981): Ecología y Paisaje. Madrid, Blume.

Lesschen, J. P., Cammeraat, L. H. y Nieman, T. (2008): "Erosion and terrace failure due to agricultural land abandonment in a semi-arid environment". Earth Surface Processes and Landforms 33, pp. 1574-1584.

Mometto, P. (1992): Lazienda agricola Barbarigo a Carpi. Venecia, Il Cardo Editore, Collana della Facoltà di Lettere e Filosofia dell'Università di Venezia.

Pausas, J. G. (2003): "The effect of landscape pattern on Mediterranean vegetation dynamics Mediterranean vegetation dynamics: A modelling approach using functional types". Journal of Vegetation Science 14, pp. 365-374.

Pérez-Cueva, A. J. (1994): Atlas climático de la Comunidad Valenciana (1961-1990). Valencia, Conselleria de Obras Públicas, Urbanismo y Transporte.

Rivas-Martínez, S. y Arnaiz, C. (1983): "Bioclimatología y vegetación en la Península Ibérica”. Pré-rapport du Colloque de Bioclimatologie Mediterraneenne: III. Montpellier.

Rackham, O. (1986): The history of the countryside. Londres, J.M. Dent.

Sereni, E. (1961): Storia del paesaggio agrario italiano. Roma, Gius. Laterzi e Figli.

Turri, E. (2002): La conoscenza del territorio. Metodologia per un'analisi storico-geografica. Venecia, Marsilio Editori.

\section{RESUMEN}

Este estudio de caso de un paisaje agrícola en un sistema de huerta tradicional de la provincia de Alicante fue llevado a cabo para desarrollar técnicas adecuadas de cuantificación y análisis del paisaje en el período 1883-2007. Los métodos utilizados se derivan del análisis de cambio en la estructura del paisaje, basado en antiguos mapas y fotografías aéreas, siendo adaptados a una escala detallada por medio de herramientas SIG. La base de datos creada para este análisis diacrónico es una pieza fundamental para obtener una clara visión de la evolución de los usos del suelo en el área de estudio. El principal objetivo de este estudio es poner de manifiesto que una aproximación a nivel de parcela, utilizando tecnologías SIG, proporciona valiosos resultados para planificar programas de conservación en paisajes agrícolas a escala local.

Palabras Clave: cambio; patrón agrícola; paisaje; cartografía; Alicante. 


\begin{abstract}
This case study of a cultural landscape in a traditional irrigated system of Alicante Province was conducted to develop appropriate techniques for quantifying and analyzing the landscape change since 1883. Methods were derived from landscape structure change analysis, based on old maps and aerial photographs, and were rendered at a high scale level using GIS tools. The created database for the diachronic GIS analysis is a key piece in order to obtain a clear view of land uses evolution in the study area. The main objective of this study is to emphasize that an approach based on a land plot level and using GIS technologies provides valuable results for planning conservation programs in cultural landscapes at local scale.
\end{abstract}

KEY WORDS: change; agricultural pattern; landscape; cartography; Alicante.

\title{
RÉSUMÉ
}

Cette étude de cas d'un paysage agricole dans un système de potager traditionnel de la province d'Alicante a été menée à bien pour développer des techniques adéquates de quantification et analyse du paysage dans la période 1883-2007. Les méthodes utilisées dérivent de l'analyse de changement dans la structure du paysage, basé sur d'anciennes cartes et photographies aériennes, en étant adaptées à une échelle détaillée au moyen d'outils SIG. La base de données créée pour cette analyse diachronique est une pièce fondamentale pour obtenir une vision claire de l'évolution des utilisations du sol dans le champ d'étude. Le principal objectif de cette étude est de mettre en évidence qu'une approximation au niveau de parcelle, en utilisant des technologies SIG, fournit de précieux résultats pour planifier des programmes de conservation dans des paysages agricoles à l'échelle locale.

MotS CLÉS: changement; modèle agricole; paysage; cartographie; Alicante. 